\title{
A new compact young moving group around V1062 Scorpii
}

\author{
Siegfried Röser ${ }^{1,2}$, Elena Schilbach ${ }^{1,2}$, Bertrand Goldman $^{3,4}$, \\ Thomas Henning ${ }^{3}$, Attila Moor ${ }^{5}$, and Aliz Derekas ${ }^{5,6}$

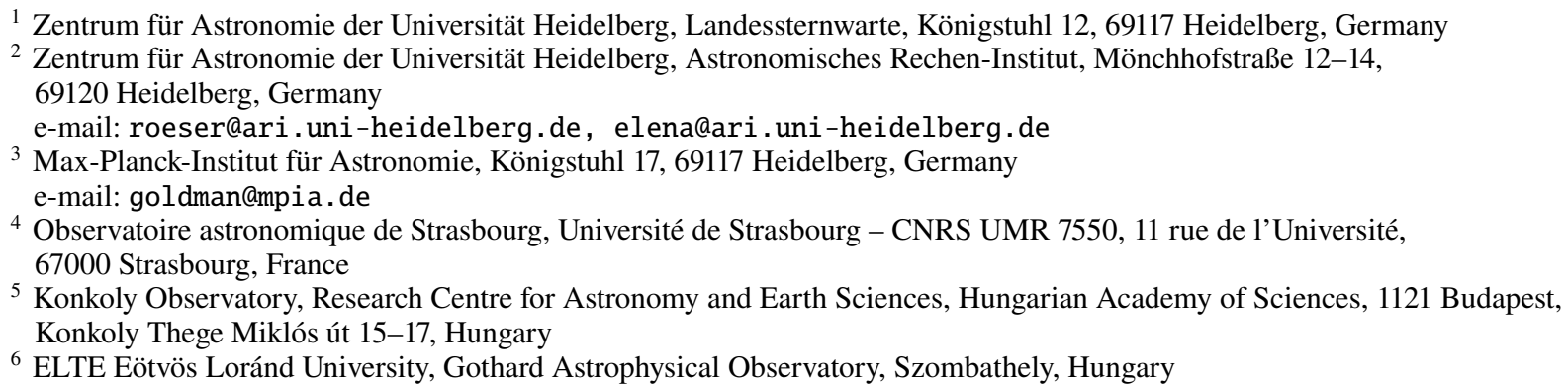

Received 31 October 2017 / Accepted 27 December 2017

\begin{abstract}
Aims. We are searching for new open clusters or moving groups in the solar neighbourhood.

Methods. We used the Gaia-TGAS catalogue, cut it into narrow proper motion and parallax slices and searched for significant spatial over-densities of stars in each slice. We then examined stars forming over-densities in optical and near-infrared colour-magnitude diagrams to determine if they are compatible with isochrones of a cluster.

Results. We detected a hitherto unknown moving group or cluster in the Upper Centaurus Lupus (UCL) section of the ScorpiusCentaurus OB association (Sco-Cen) at a distance of $175 \mathrm{pc}$ from the Sun. It is a group of 63 comoving stars of less than 10 to about $25 \mathrm{Myr}$ in age. For the brightest stars that are present in the Gaia-TGAS catalogue, the mean difference between kinematic and trigonometric distance moduli is $-0.01 \mathrm{mag}$ with a standard deviation of $0.11 \mathrm{mag}$. Fainter cluster candidates are found in the HSOY catalogue, where no trigonometric parallaxes are available. For a subset of our candidate stars, we obtained radial velocity measurements at the MPG/ESO $2.2 \mathrm{~m}$ telescope in La Silla. Altogether we found 12 members with confirmed radial velocities and parallaxes, 31 with parallaxes or radial velocities, and 20 candidates from the convergent point method. The isochrone masses of our 63 members range from 2.6 to $0.7 M_{\odot}$.
\end{abstract}

Key words. parallaxes - proper motions - stars: formation - open clusters and associations: general open clusters and associations: individual: Upper Centaurus Lupus

\section{Introduction}

For more than a century, the Scorpius-Centaurus OB association (Sco-Cen) has been studied as a moving group of early-type stars. Blaauw (1964) divided the huge nearby association into the following three subgroups: Upper Scorpius; Upper Centaurus Lupus (UCL), also known as Sco OB2_3; and Lower Centaurus Crux. After the ESA HIPPARCOS mission, accurate proper motions and trigonometric parallaxes became available for many early-type stars in Sco-Cen. This material was used by de Zeeuw et al. (1999) for their fundamental paper on OB associations.

Among the HIPPARCOS objects in the UCL subgroup of ScoCen, de Zeeuw et al. (1999) identified 221 members in this field, which have a mean distance of $140 \pm 2 \mathrm{pc}, 66 \mathrm{~B}, 68 \mathrm{~A}, 55$ F, $25 \mathrm{G}$, six $\mathrm{K}$, and one M-type star. They also noted that the star-forming regions (SFRs) associated with the Lupus clouds contained several tens of low-mass pre-main-sequence stars with estimated ages of $\approx 3 \mathrm{Myr}$ (see the references in de Zeeuw et al. 1999). These SFRs cover most of UCL, except a small area in the south-eastern corner of UCL. We found a compact, young moving group of stars in this area while searching for open clusters in the TGAS catalogue (Michalik et al. 2015; Lindegren et al. 2016).
The first Gaia data release (Gaia-DR1; Gaia Collaboration 2016), and especially the publication of TGAS with its highprecision proper motions and trigonometric parallaxes fostered work on open clusters and moving groups by a number of authors. Without aiming for completeness we mention here the work performed by Gaia Collaboration (2017) on nearby open clusters and of Randich et al. (2018) who, combining information from Gaia-DR1 and the Gaia-ESO Survey (Gilmore et al. 2012) data, derive new distances and ages of eight nearby open clusters. Oh et al. (2017) used the TGAS catalogue to search for highconfidence comoving pairs of stars. In doing so these authors found some ten thousand comoving star pairs with separations as large as $10 \mathrm{pc}$. They also found larger groups corresponding to known open clusters and $\mathrm{OB}$ associations and several new comoving groups.

Studying the structure and behaviour of moving groups may shed light on the process of star formation in associations. We are guided by the statement by Larson (2002) in his summary during the conference "Modes of Star Formation and the Origin of Field Populations": "When associations disperse, they retain some kinematic coherence because their internal motions are relatively small, and they then become moving groups or streams. 


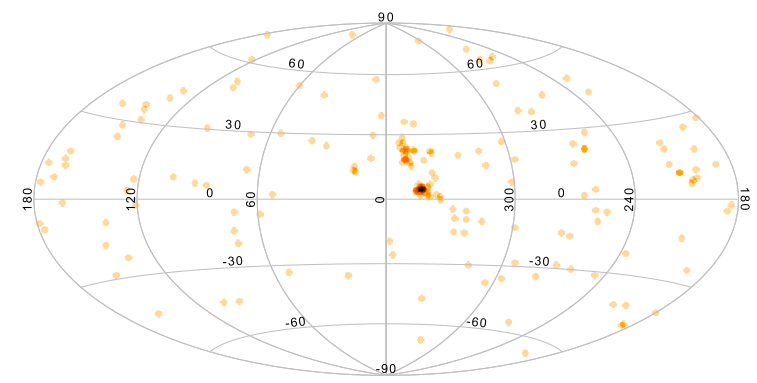

Fig. 1. Sky map (in Galactic coordinates) showing the stars from the TGAS catalogue with parallaxes between 5 and 10 mas and in the proper motion slice around $\left(\mu_{\alpha} \cos \delta, \mu_{\delta}\right)=(-12,-22)$ mas $\mathrm{yr}^{-1}$. For further information on the selection see text.

The weak-lined T Tauri stars found by X-ray observations that were discussed at this meeting probably represent some of the remnants of associations that are just now dissolving into the field and becoming moving groups". So, it was worthwhile to study our new moving group in more detail as all ingredients discussed by Larson are in place; these include an association, a good number of $\mathrm{T}$ Tauri and weak-line $\mathrm{T}$ Tauri stars, and a moving group.

In this paper we describe in Sect. 2 how we detected this group, as well as the spectroscopic follow-up measurements we carried out with the FEROS instrument at the MPG/ESO $2.2 \mathrm{~m}$ telescope in La Silla to obtain radial velocities (RVs) and stellar parameters. In Sect. 3 we provide a discussion of the properties of the newly found group. Then, in Sect. 4 we compare our new group with the star-forming areas in the other parts of UCL, and a short summary concludes the paper.

\section{Detection of the V1062 Sco moving group}

\subsection{Astrometric detection}

After the first Gaia data release in September 2016, we performed a cluster search programme similar to that described in Röser et al. (2016), but with TGAS replacing the Tycurat catalogue that we used originally. In brief the method consists in cutting the TGAS catalogue into slices as follows: In proper motion space we place a grid with grid points $(i, k)$ at each inte-

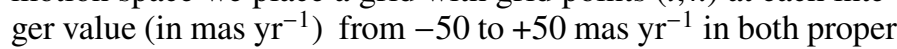
motion components. A slice at $(i, k)$ contains all stars for which $\left(\mu_{\alpha} \cos \delta-i\right)^{2}+\left(\mu_{\delta}-k\right)^{2} \leq 1.5^{2}\left(\text { mas yr }^{-1}\right)^{2}$ holds. For each slice, we then check the distribution of its stars over the sky and search for possible over-densities that can be considered as candidates of clusters or moving groups.

The availability of the TGAS parallaxes allows us to improve the effectivity of the method by cutting the catalogue into parallax slices as well. Since we are especially interested in finding unknown clusters in the vicinity of the Sun, we consider a parallax slice between 5 and 10 mas that contains the stars between 100 and $200 \mathrm{pc}$ from the Sun. Compared to the full TGAS sky with some 2 million stars this parallax slice only includes 160000 stars. The latter is then cut into proper motion slices. In Fig. 1 we show a sky map in Galactic coordinates of the proper motion slice $(i, k)=(-12,-22)$ mas $\mathrm{yr}^{-1}$ that only contains 159 stars. We found a hitherto unknown overdensity containing 25 stars (of 159) in a radius of $2.5^{\circ}$ around $(l, b)=\left(343^{\circ} .6,+4^{\circ} .3\right)$ in UCL. The fainter filamentary structure north of the over-density may also be a substructure in Sco-Cen, but we have not investigated this structure yet. Our

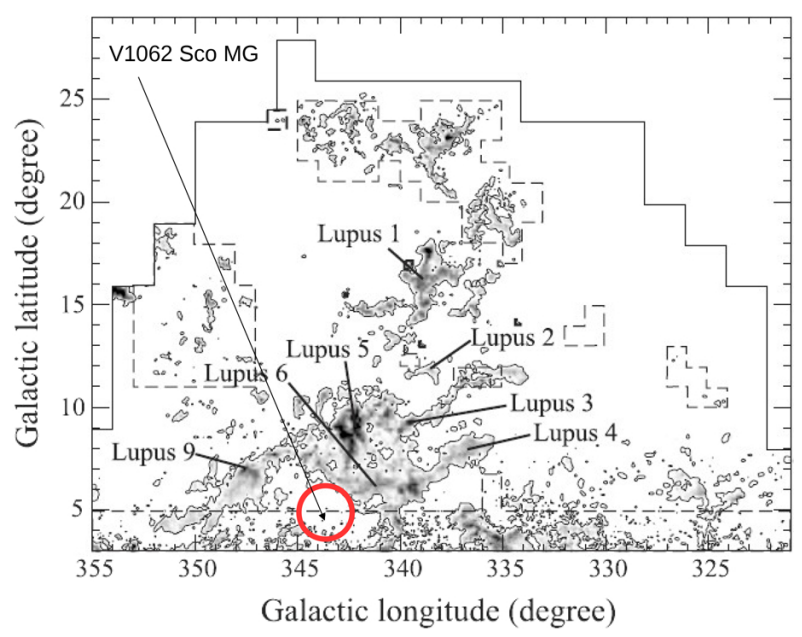

Fig. 2. Location of the V1062 Sco moving group (red circle) overlaid on the CO map from Tachihara et al. (2001). The radius of the circle is $2.75 \mathrm{deg}$; or $8.4 \mathrm{pc}$ at a distance of $175 \mathrm{pc}$ and roughly corresponds to the extent of the group.

newly found over-density in the south-eastern corner of UCL occupies an area that is void on the ${ }^{12} \mathrm{CO}$ map from Tachihara et al. (2001). We show its location in detail in Fig. 2. Also, the trigonometric parallaxes of these 25 stars show a narrow range between 5.3 and 6.3 mas in TGAS (cf. Fig. 7), we henceforth refer to this over-density of comoving stars as the V1062 Sco moving group (V1062 Sco MG) according to its prominent member V1062 Sco, postponing the discussion if V1062 Sco is a true member or not to Sect. 3.4. Parallel to our finding of this new moving group this object has been detected in the above-mentioned paper by Oh et al. (2017) as their "Group 11", but without further discussion.

\subsection{Radial velocities and stellar parameters}

In order to get additional confirmation for the existence of the V1062 Sco MG, we carried out FEROS observations at the MPG/ESO $2.2 \mathrm{~m}$ telescope in July 2017 and determined RVs for a subset of possible moving group candidates. The fibre-fed, high-resolution (48000) cross-dispersed optical spectrograph, FEROS, is mounted on the MPG/ESO $2.2 \mathrm{~m}$ telescope in La Silla (Kaufer et al. 1999). FEROS has two set-ups: one of its two fibres points to the science target, and the second points either to a calibration lamp for better wavelength calibration, or to the sky. For brighter targets, we used both set-ups and for fainter targets we only used the sky set-up. We found that for our observations, the wavelength calibration errors are a minor contributor to the error budget. The observing log is given in Table A.4.

To reduce the spectra we used the CERES pipeline (Brahm et al. 2018), a reduction and extraction pipeline dedicated to échelle spectrographs. In a nutshell, for each of our three observing nights, CERES identifies the calibration files and obtains a master bias and dark frames through median filtering. The flat field is not removed at this stage but instead the blaze function obtained from continuum lamp frames is divided after extraction, which also corrects the column-averaged pixel-to-pixel variations of the CCD. The pipeline then identifies the order traces and fits them with a high-order polynomial; it measures the scattered light in the inter-order zones, interpolating over the spectra and performs a 2D median filtering of the resulting map. After subtraction of the scattered light, the spectra are optimally extracted. The spectra are wavelength-calibrated using the ThAr 
lamp spectra, observed either simultaneously with the target or during the afternoon.

The CERES pipeline measures RVs by cross-correlating the spectra with binary masks indicating the narrow absorption lines, following Mayor et al. (2003). As CERES is optimized for late-type stars in its standard configuration, it provides masks corresponding to G2, K5, and M2 spectral types. Measurements of early-type stars is notoriously difficult as their spectra exhibit few absorption lines that are considerably broadened by stellar rotation. In the following we deal only with spectra of candidates with effective temperatures smaller than $7000 \mathrm{~K}$. The uncertainties on the RV is determined as a function of the signal-to-noise ratio $(\mathrm{S} / \mathrm{N})$ at the $\mathrm{Mg}$-triplet wavelength and the width of the cross-correlation function $(\mathrm{CCF})$; the parameters are determined from a Monte Carlo simulation using degraded high S/N spectra. The measurements are reported in Table A.4. For targets with multiple observations we report the weighted average and the error on the mean of all the available measurements and list these stars in Table A.5. The dispersion of these individual observations roughly confirms the uncertainties derived from the S/Ns.

To obtain the stellar parameters, we applied the ZASPE (Brahm et al. 2016) pipeline. The pipeline is optimized to use the spectral library of Brahm et al. (2016), created in order to reduce the biases in the stellar parameters with respect to the measurements of SWEET-Cat (Santos et al. 2013). This library covers a range of stellar temperatures up to $7000 \mathrm{~K}$. The precision of the parameters can be estimated from the repeated observations of some targets, typically a few 0.1 dex in $\log g, 0.1$ dex in $[\mathrm{Fe} / \mathrm{H}]$, $200 \mathrm{~m} \mathrm{~s}^{-1}$ in $v \sin i$, a few dozen Kelvin in $T_{\text {eff }}$. Average values of these parameters are given in Table A.5. Our cluster members or candidates $(\mathrm{m}(4 \mathrm{~d})$ to $\mathrm{m}(6 \mathrm{~d})$; see Sect. 3.1) have a mean metallicity of 0.04 dex and have a spread of $0.1 \mathrm{dex}$. Their rotational velocity ranges from 5 to $80 \mathrm{~km} \mathrm{~s}^{-1}$. Only one cluster candidate member shows $\mathrm{H}_{\alpha}$ emission, namely star 25, which also displays (stronger) absorption. This general lack of emission, indicating no accretion, is consistent with the age range determined from the isochrones (see Sect. 3.2).

\subsection{Phase space coordinates}

For 11 out of the 25 kinematic candidate stars mentioned in Sect. 2.1 we obtained well-determined RVs with formal errors less than $1 \mathrm{~km} \mathrm{~s}^{-1}$. Their RVs pile up between 1.0 to $+3.6 \mathrm{~km} \mathrm{~s}^{-1}$ and give $\mathrm{RV}_{\text {mean }}=+2.24 \pm 0.85 \mathrm{~km} \mathrm{~s}^{-1}$. Moreover, their parallaxes turned out to be within a narrow range between 5.4 and 6.2 mas, corresponding to a mean distance from the Sun of $175 \pm 7$ pc. For these stars we now have the complete set of $6 \mathrm{D}$ phase space coordinates based on direct observations. Hence we compute the mean space coordinates and velocities of the group in barycentric Galactic coordinates to be

$$
(X, Y, Z, U, V, W)=(167.20,-49.14,13.44,-3.80,-19.96,-4.06) .
$$

Here the $X$-axis points to the Galactic centre, the $Y$-axis to the direction of Galactic rotation, and the $Z$-axis points to the Galactic North Pole. The corresponding components of the space velocity are $U, V, W$. The standard deviation in the three components of the space velocity is rather small $\left(\sigma_{U}, \sigma_{V}, \sigma_{W}\right)=$ $(0.7,0.9,0.3) \mathrm{km} \mathrm{s}^{-1}$. The individual residuals in velocity space do not show systematic effects such as rotation or expansion. The standard deviation sets an upper limit of $1 \mathrm{~km} \mathrm{~s}^{-1}$ for the $1 \mathrm{D}$ velocity dispersion of the group, and we show in the discussion in Sect. 3.1 that this is large compared to the formal error at least for that component of the tangential velocity, which is perpendicular to the direction to the convergent point.

\section{Properties of the V1062 Sco moving group}

We can use the phase space coordinates (1) of the V1062 Sco MG to perform a search of its members. This can be carried out on the basis of the convergent point $(\mathrm{CP})$ method we applied to find members in the Hyades cluster (Röser et al. 2011; Goldman et al. 2013). In case of the V1062 Sco MG, we extended the search to a distance of $10 \mathrm{pc}$ around the centre $(X, Y, Z)=(167.20,-49.14,13.44)$ from $(1)$. Using the proper motions given in TGAS, the method predicts kinematic parallaxes $\varpi_{\text {kin }}$, and the velocity components in the tangential plane parallel and perpendicular to the direction of the convergent point. In the ideal case of an exactly parallel space motion the perpendicular component has to be zero. Taking into account a possible internal velocity dispersion and the accuracy of the input data, we allowed a maximum of $3 \mathrm{~km} \mathrm{~s}^{-1}$ for the perpendicular velocity component. With these restrictions we found 35 TGAS stars as kinematic candidates of the moving group. Further we required that the difference between the predicted kinematic parallaxes $\varpi_{\text {kin }}$ and the observed trigonometric parallaxes $\varpi_{\text {trig }}$ from TGAS to be smaller than the mean error of this difference, i.e.

$\left|\varpi_{\text {kin }}-\varpi_{\text {trig }}\right|<\left(\sigma_{\varpi_{\text {kin }}}^{2}+\sigma_{\varpi_{\text {trig }}}^{2}+0.3^{2}\right)^{1 / 2}$,

where 0.3 mas was taken as a possible systematic error of TGAS trigonometric parallaxes (Lindegren et al. 2016). The application of the restriction (2) deletes two candidates, so we finally retained 33 kinematic members in our group confirmed by their trigonometric parallaxes. We publish them in Table A.1.

In order to find fainter candidates of the V1062 Sco MG, we also used the HSOY catalogue (Altmann et al. 2017). This catalogue was constructed using Gaia-DR1 positions as an additional epoch together with PPMXL positions and proper motions to obtain formally improved proper motions. Although HSOY may have significant systematic proper motion errors over the sky, these should be unimportant for work in small areas of some tens of square degrees. We restricted our search within HSOY to $G \leq 14$ mag that yielded 17 million stars; this subset is called HSOY14 in the following. This restriction was driven by the fact that the moving group is located south of declination $-30^{\circ}$ and the quality of the proper motions in HSOY for fainter stars deteriorates in this quarter of the sky. Using the convergent point method with the phase space coordinates (1) we derived kinematic parallaxes, which, however, cannot yet be verified by the trigonometric parallax observations. So, as in the case of the TGAS stars, the distance moduli and absolute magnitudes are derived from kinematic parallaxes. The CP method yielded 149 candidates. We then usually discard background stars that appear below the ZAMS and only retain stars that fulfil $M_{G} \leq 3 \times(G-J)+2$ in the $M_{G}, G-J$ colour-magnitude-diagram (CMD). Forty stars are discarded by this criterium; these are all redder than $(G-J)=1.0$. Since HSOY 14 contains TGAS as a subset, we had to discard the above-mentioned 35 TGAS stars from the remaining 109 stars. It turns out, however, that from the remaining 74 HSOY 14 stars 9 were also in the TGAS catalogue. Their proper motions differ slightly in the HSOY 14 and TGAS catalogues, and this was a reason why they were not selected as TGAS candidates. Since the formal precision of the proper motions was a factor of almost two better for these stars in 
HSOY14 compared to TGAS, we kept them as kinematic candidates. Moreover, 7 out of 9 stars fulfilled the condition (2). These 9 stars are given in Table A.2.

As the remaining 65 faint HSOY 14 candidates were only selected on the basis of their HSOY14 proper motions, we checked the data quality by cross-matching the stars with UCAC5 (Zacharias et al. 2017) for consistency. We adopted this approach for the following reasons: The parent catalogue of HSOY is PPMXL, which itself descends from USNO-B1.0 (Monet et al. 2003). South of $-30^{\circ}$ declination USNO-B1.0 is of poor quality, as the epoch difference between the first and second epoch is low in the south, and a possible mismatch leads to grossly erroneous proper motions that may be handed over to HSOY. A comparison with a completely independent source such as UCAC5 removes this problem. So, we only retain those stars whose difference in proper motions between HSOY and UCAC5 is within $1 \sigma$ of the formal error of this difference. Thirty-two stars of 65 survived this check and are given in Table A.3.

\subsection{Membership}

Because of the selection procedure applied, our sample of kinematic candidates includes stars of different membership probability. The quality of membership is based mainly on the observations available for candidates that are consistent with the phase-space coordinates of the V1062 Sco MG defined by (1). If, as in the case of HSOY, only four parameters $\left(\alpha, \delta, \mu_{\alpha}\right.$, $\left.\mu_{\delta}\right)$ are available and consistent with (1), we call these candidates $\mathrm{m}(4 \mathrm{~d})$ members. The $\mathrm{CP}$ method predicts the parallax and $\mathrm{RV}$ of candidate members. Both predictions have to be verified by observations. If no trigonometric parallaxes are available we can only make a negative selection by testing if the predicted absolute magnitudes are compatible with allowed stellar loci in CMDs. By doing this, we indeed classified a few $\mathrm{m}(4 \mathrm{~d})$ candidates as non-members because of their location in CMDs (see Sect. 3.2)

However, the majority of our candidates had measurements of trigonometric parallaxes $\left(\varpi_{\text {trig }}\right)$ or/and RVs. If one of the quantities RV, or $\varpi_{\text {trig }}$ is measured and consistent with (1), we called the star a $\mathrm{m}(5 \mathrm{~d})$ member since its five coordinates of the $6 \mathrm{D}$ phase space coincide with the assumption of their membership in the V1062 Sco MG. If RV and $\varpi_{\text {trig }}$ were measured and fulfil (1), we call them $\mathrm{m}(6 \mathrm{~d})$ members. On the other hand, if one of the measurements was inconsistent with (1), we rejected this star as a member and call it a non-member (nm).

In our total sample of 76 kinematic candidates of the V1062 Sco MG, we found $12 \mathrm{~m}(6 \mathrm{~d})$ members. These are fully confirmed members and their existence proves the authenticity of the moving group. Also the $31 \mathrm{~m}(5 \mathrm{~d})$ members are very probable candidates, as their measured $\varpi_{\text {trig }}$ confirms the predicted $\varpi_{\text {kin }}$ from the CP method, or, in four cases, the measured RV confirm the predicted RV. The other $20 \mathrm{~m}(4 \mathrm{~d})$ candidates await confirmation by RV and $\varpi_{\text {trig }}$ measurements. Finally, in three cases the predicted parallaxes were not confirmed by the observed parallaxes, in one case RV was inconsistent, and one star was rejected owing to both inconsistent parallax and RV. At this point we want to emphasize that, using the photometric criteria, we also identified $8 \mathrm{~m}(4 \mathrm{~d})$ candidates as non-members (see the Sect. 3.2). An overview of the membership quality in the V1062 Sco MG is given in Table 1.

The stars in our V1062 Sco MG are remarkably comoving. Although we allowed the component of the tangential velocity perpendicular to the direction to the convergent point to
Table 1. Overview of the membership quality in the V1062 Sco MG.

\begin{tabular}{crrrr}
\hline \hline Membership & Table A.1 & Table A.2 & Table A.3 & All \\
\hline $\mathrm{m}(6 \mathrm{~d})$ & 11 & 1 & - & 12 \\
$\mathrm{~m}(5 \mathrm{~d})\left[\varpi_{\text {trig }} /\right.$ no RV $]$ & 22 & 5 & - & 27 \\
$\mathrm{~m}(5 \mathrm{~d})\left[\mathrm{RV} / \mathrm{no} \varpi_{\text {trig }}\right]$ & - & - & 4 & 4 \\
$\mathrm{~m}(4 \mathrm{~d})$ & - & - & 20 & 20 \\
$\mathrm{~nm}$ & 2 & 3 & 8 & 13 \\
\hline All & 35 & 9 & 32 & 76 \\
\hline
\end{tabular}

Notes. In Col. 1 we show various membership qualities as explained in the text, Col. 2 refers to TGAS stars from Table A.1, Col. 3 to HSOY stars with TGAS parallaxes (Table A.2), and Col. 4 to HSOY stars (Table A.3). Finally, in Col. 5 we give the total number of stars with the respective membership quality.

$\pm 3 \mathrm{~km} \mathrm{~s}^{-1}$, we find an overall standard deviation as small as $1 \mathrm{~km} \mathrm{~s}^{-1}$ around the expectation value of zero. At $175 \mathrm{pc}$ this

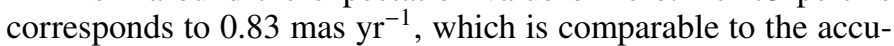
racy of the TGAS proper motions. The corresponding scatter of the $12 \mathrm{~m}(6 \mathrm{~d})$ members amounts to only $0.3 \mathrm{~km} \mathrm{~s}^{-1}$ or less than 0.3 mas $\mathrm{yr}^{-1}$ comparable to the accuracy of the best TGAS stars. As the perpendicular component of the tangential velocity is a measure of the actual 1D velocity dispersion in a cluster (moving group), we may safely conclude that it should be less than $1 \mathrm{~km} \mathrm{~s}^{-1}$.

\subsection{Colour-magnitude diagrams}

All stars of the V1062 Sco MG as selected above have 2MASS (Skrutskie et al. 2006) photometry and Gaia DR1 (Gaia Collaboration 2016) $G$ magnitudes. We present them in the colour-magnitude diagrams (CMDs) $M_{G}$ versus $G-J$ in Fig. 3 and $M_{K_{\mathrm{S}}}$ versus $J-K_{\mathrm{S}}$ in Fig. 4. The magnitudes on the ordinate are absolute magnitudes and the colours on the abscissae are intrinsic colours in both diagrams. For the distance moduli we used the $\varpi_{\text {kin }}$, first because they are available for all the stars in the 3 tables, and second because, within the TGAS precision budget, they are formally more precise than the $\varpi_{\text {trig }}$. In any case the mean difference between the distance moduli from $\varpi_{\text {trig }}$ and $\varpi_{\text {kin }}$ is -0.01 mag with a standard deviation of $0.11 \mathrm{mag}$, and therefore hardly visible on the plots. We also do not show error bars for the absolute magnitudes. For TGAS stars the median of the uncertainties in distance modulus is $0.1 \mathrm{mag}$ based on kinematic and 0.17 mag based on trigonometric parallaxes. For HSOY stars the median is $0.26 \mathrm{mag}$. All these quantities give error bars that are smaller than the size of the symbols. The reddening $E(B-V)$ was determined from photometric data on TGAS members since their actual distances are sufficiently well known from the trigonometric parallaxes. We obtained a value of $0.065 \mathrm{mag}$ for $E(B-V)$ from the loci of the star in the three CMDs, i.e. $M_{G}$ versus $G-J, M_{K_{\mathrm{S}}}$ versus $J-K_{\mathrm{S}}$, and $M_{V}$ versus $B-V$.

Padova-2.8 solar metallicity isochrones (Bressan et al. 2012) for $\log t=6.6,7.4$ and 8.0 are also shown in Figs. 3 and 4. From the distribution of the stars in both CMDs we estimate that the masses of our members range between 2.6 to $0.7 M_{\odot}$.

It is conspicuous in both CMDs that the stars do not follow a unique isochrone, but are mainly located between isochrones of 4 and $25 \mathrm{Myr}$, especially those with spectral types later than about F0 $\left(J-K_{\mathrm{S}}>0.2\right)$. This spread cannot be compensated by the inaccuracy of photometric data: a typical $r m s$ error is about 


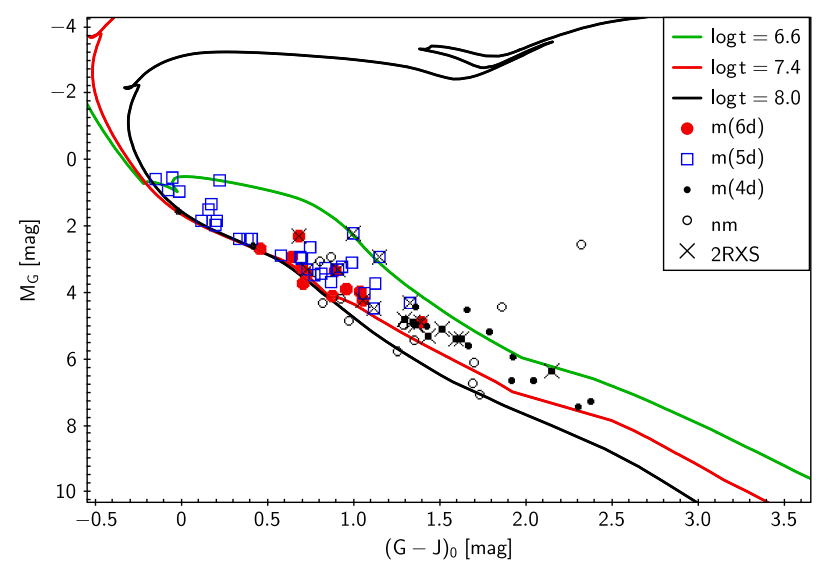

Fig. 3. Colour-magnitude diagram $M_{G}$ vs. $(G-J)_{0}$ of stars in the V1062 Sco MG. The symbols for the members of different membership quality are shown in the legend. Crosses indicate stars that were observed by the ROSAT satellite (2RXS, see text). Also plotted are three Padova-2.8 solar metallicity isochrones for $\log t=6.6,7.4$ and 8.0.

$0.025 \mathrm{mag}$ for $J, K_{\mathrm{S}}$, and $0.01 \mathrm{mag}$ for $G$ magnitudes. Also, binarity can alleviate the age discrepancy, but not completely remove it. A possible explanation can be that the stars have formed over a period of $20 \mathrm{Myr}$, but are still moving together with the same space velocity. We note that the V1062 Sco MG is embedded in the Sco-Cen OB association, where many small SFRs are present (see the discussion in the next section), but, to our knowledge, not one such region is found that is as nicely comoving and well populated as the V1062 Sco MG.

We also tried to recover our stars in other sky surveys to get further indication on their youth. We found that eight TGAS stars ( $3 \mathrm{~m}(6 \mathrm{~d}), 5 \mathrm{~m}(5 \mathrm{~d}))$ and nine HSOY14 stars $(1 \mathrm{~m}(5 \mathrm{~d}), 8 \mathrm{~m}(4 \mathrm{~d}))$ have ROSAT-2RXS (Boller et al. 2016) counterparts, but none of these is measured by Galex (Morrissey et al. 2007) as our moving group is outside the area that Galex observed.

As we mentioned above, the convergent point method predicts the parallaxes of the kinematic members. In contrast to TGAS candidates, we cannot yet verify the predictions for HSOY stars by measurements. Therefore, some few HSOY kinematic candidates may happen to be non-members of the V1062 Sco MG. We can decrease a possible contamination by checking the consistency of predicted parallaxes and photometric distances of $\mathrm{m}(4 \mathrm{~d})$ candidates. To be on a safe side, we marked a candidate as a non-member when it is located outside the area bordered by the isochrones $\log t=6.6,7.4$ in at least one of the CMDs $\left(M_{G}\right.$ versus $G-J, M_{K_{\mathrm{S}}}$ versus $\left.J-K_{\mathrm{S}}\right)$. Five of eight rejected candidates indeed seemed to be background or foreground stars, whereas three others were probably main-sequence stars at the distance of the V1062 Sco MG. In this case they may be comoving field stars older than $100 \mathrm{Myr}$, which are likely not connected to the young V1062 Sco MG, i.e. contaminants.

\subsection{Estimate of the contamination}

To estimate the number of field star contaminants that may enter our sample, we ran a Besançon simulation of the area around the cluster centre, obtaining a mock catalogue of field objects with $6 \mathrm{D}$ parameters. The Besançon simulation was performed over $40 \mathrm{sq}$. deg. with default parameters. The area was centred at $l=343^{\circ} .7, b=+4.3$ and a constant extinction of $A_{V}=$ $1.2 \mathrm{mag} \mathrm{kpc}^{-1}$ was added. This agrees with $A_{V}=0.2 \mathrm{mag}$ at 175 pc, i.e. at the mean distance of the V1062 Sco MG, where

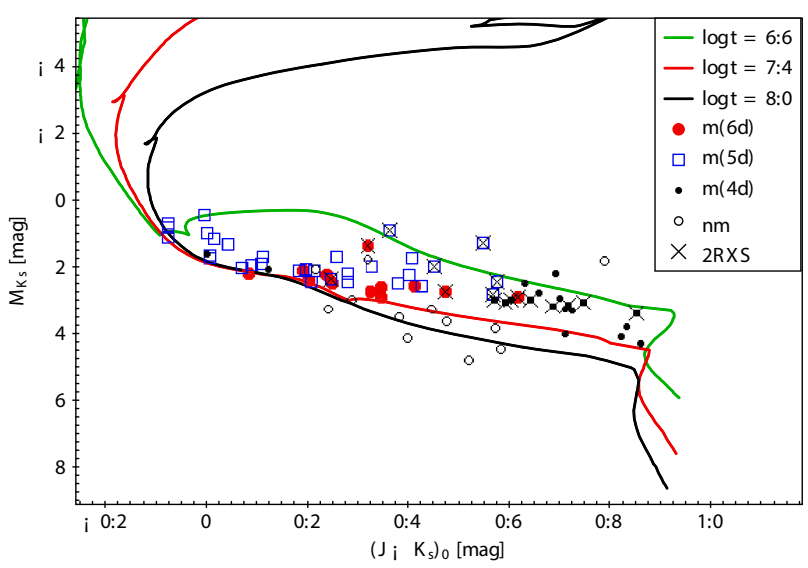

Fig. 4. Colour-magnitude diagram $M_{K_{\mathrm{S}}}$ vs. $\left(J-K_{\mathrm{S}}\right)_{0}$ of stars in the V1062 Sco MG. The symbols for the members of different membership quality are shown in the legend. Crosses indicate stars that were observed by the ROSAT satellite (2RXS, see text). Also plotted are three Padova-2.8 solar metallicity isochrones for $\log t=6.6,7.4$ and 8.0.

we estimated $E(B-V)$ to be 0.065 mag. We spread the mock positions over the whole area of $40 \mathrm{sq}$. deg. assuming a uniform distribution on the sky; the line-of-sight distance was determined by the Besançon simulator. The simulated cartesian parameters were converted into the observables, and we added Gaussian

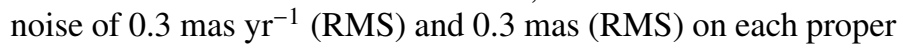
motion component and the parallax, respectively. We also converted the Besançon Cousins photometry into the Gaia $\mathrm{G}$ system using the Jordi et al. (2010) colour transformation. Finally, to improve the statistics, we repeated the simulation ten times.

As for the real TGAS and HSOY14 stars, we ran the CP method around the phase-space coordinates (1) for this simulation catalogue enlarged ten times. In Fig. 5 we show the simulated stars that came out from the CP method as kinematic candidates, i.e. $\mathrm{m}(4 \mathrm{~d})$ members. In total, there are 64 stars in the area between the isochrones for $\log t=6.6$ and $\log t=7.4$. So, in fact, we expect a number of 7 (6.4) stars from the Besançon model to fall into the CMD area occupied by the stars from the V1062 Sco MG. In the following we give a worst case (maximum) estimate of the contamination: From our 68 kinematic candidates from Table 1 in the CMD area we had to discard already 5 as non-members based on discordant parallaxes or radial velocities. So, the maximum contamination among the remaining 63 is two, or $3 \%$. In reality the contamination is much smaller for the $\mathrm{m}(5 \mathrm{~d})$ or $\mathrm{m}(6 \mathrm{~d})$ members. For instance, if we take into account the simulated parallaxes of the 6.4 contaminants, we find only 0.7 that should fall into the CMD area in Fig. 5.

\subsection{Individual stars}

The star $\mathrm{V}^{*}$ V1062 Sco (star 7 of our sample) is a variable of $\alpha_{2}$ CVn type. Its TGAS parallax makes it an m(5d) member in our terminology. In the literature we found a RV measurement of $13.00 \pm 5.80 \mathrm{~km} \mathrm{~s}^{-1}$ from Levato et al. (1996). This RV is almost $2 \sigma$ off the predicted RV. However, in case of a rotationally variable stars it is hard to extract its line-of-sight velocity. Therefore we keep this star as an $\mathrm{m}(5 \mathrm{~d})$ member.

Star 5 (HD 149551) and star 18 (HD 150372) are found in Mamajek et al. (2002). Depending on different pMS isochrones the authors estimate an age between 4 and 11 Myr and a mass between 1.5 and $1.7 M_{\odot}$ for star 5 , and an age between 4 and $7 \mathrm{Myr}$ and a mass between 1.8 and $1.9 M_{\odot}$ for star 18 . Star 9 


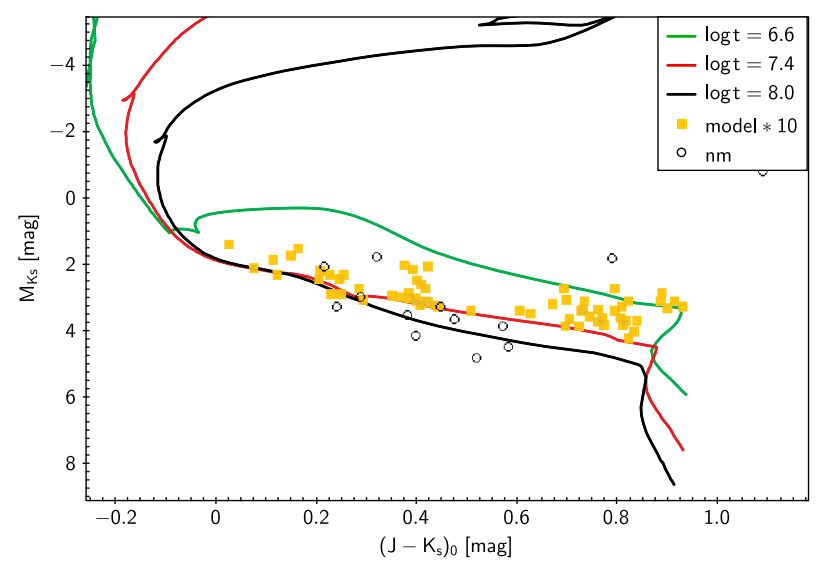

Fig. 5. Colour-magnitude diagram $M_{K_{\mathrm{S}}}$ vs. $\left(J-K_{\mathrm{S}}\right)_{0}$ of stars in the area of the V1062 Sco MG obtained from a simulation based on the Besançon model (10 times enhanced). Again three Padova-2.8 solar metallicity isochrones for $\log t=6.6,7.4$ and 8.0 are also given. Also plotted are the 13 non-members from Figs. 3 and 4 and Table 1.

(HD 149777) was rated an "active subgiant" by Mamajek et al. (2002) and hence rejected as a young member in the Sco-Cen association. However we keep it as a $\mathrm{m}(5 \mathrm{~d})$ member because of its measured trigonometric parallax. The preliminary velocity measurement $\left(R V=+0.6 \pm 7.8 \mathrm{~km} \mathrm{~s}^{-1}\right)$ for this very rapidly rotation $\operatorname{star}\left(v_{\text {rot }} \approx 200 \mathrm{~km} \mathrm{~s}^{-1}\right)$ confirms its membership. Nevertheless the uncertainty of this measurement is rather large.

Star 30 of our sample (HD 152407) is mentioned in SIMBAD as a member in the cluster Trumpler $24(\operatorname{Tr} 24)$. It has number 160 in the catalogue of $U, B, V$ measurements in Tr 24 by Heske \& Wendker (1984). According to Heske \& Wendker, TR 24 has a distance of $2300 \mathrm{pc}$, and they rate (HD 152407) as a post-MS member from their photometry and give its spectral type of B5Ib. However, its trigonometric parallax of 5.48 mas clearly rules it out as a member in $\operatorname{Tr} 24$, and sets doubts on its luminosity class Ib.

Star 141 of our sample is 2MASS J16394731-3920404, also known as [SZB2012] 86 from Song et al. (2012). These authors quote a distance of $99 \mathrm{pc}$, photometrically estimated from an empirical $10 \mathrm{Myr}$ isochrone on an $M_{K_{\mathrm{S}}}$ versus $V-K_{\mathrm{S}} \mathrm{CMD}$. They give a typical uncertainty of about $30 \%$ for their determination. This star is also found in Fuhrmeister \& Schmitt (2003) in their catalogue of X-ray variable stars as No. 844. They determine its spectral type to be MOVe and rate it as a flare star. We keep this star as an $\mathrm{m}(4 \mathrm{~d})$ candidate because of its kinematic distance of $175 \mathrm{pc}$.

Star 35 (HD 151738) is a spectroscopic binary with a difference in RV of about $100 \mathrm{~km} \mathrm{~s}^{-1}$. Because we do not know the mass ratio, we cannot use the measurement to constrain its membership.

Given the age range in V1062 Sco MG, we expect no detection of protoplanetary disks and very few detections of warm debris disks (Cieza et al. 2010). However, using VOSA (Bayo et al. 2008) we reveal a significant infrared excess in the WISE (Cutri \& et al. 2012) bands for six targets: star 107 in W1 $(3.4 \mu \mathrm{m})$ redwards, stars 22,124 , and 143 in $\mathrm{W} 3(12 \mu \mathrm{m})$ and W4 $(22 \mu \mathrm{m})$, and stars 26 and 59 in W4 only.

- Visual inspection of the relevant WISE images showed that stars 22 (HD 150532), 124 (2MASS 16375854-3933221), and 143 are associated with nebulosity both in W3 and W4 bands. Such complex backgrounds can lead to spurious detections (e.g. Cruz-Saenz de Miera et al. 2014). Actually we found no sources at the position of the stars in the W4

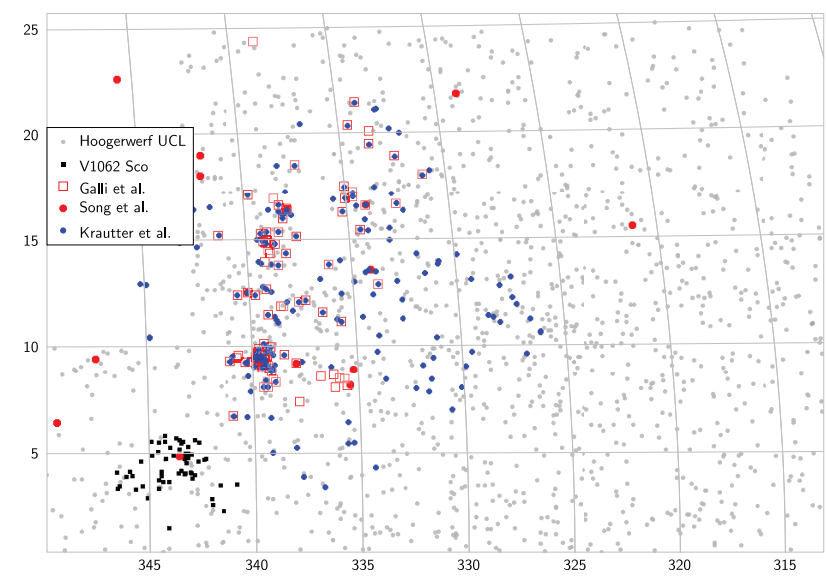

Fig. 6. Area of UCL in Galactic coordinates. The extent of UCL is outlined by the distribution of the stars from Hoogerwerf (2000; grey dots). Our 63 members of the V1062 Sco MG are shown as black squares. Young stars from other authors in the UCL area are shown with the symbols indicated on the map.

band images. The W3 band excess detections should also be taken carefully given the nebulosity.

- Star 107 (2MASS 16425498-4233057) was observed with the Spitzer Space Telescope using the IRAC instrument in the 3.6, 4.5, 5.8, and $8.0 \mu \mathrm{m}$ bands. These photometric data were taken from the Spitzer Enhanced Imaging Products catalogue $\left(\mathrm{SEIP}^{1}\right)$. In contrast to WISE observations, the analysis of IRAC data shows no evidence for excess between 3.6 and $8.0 \mu \mathrm{m}$. The reasons of these discrepancies are not yet clear.

- The IR excess of star 26 (HD 151109) was also detected by Rizzuto et al. (2012) and Chen et al. (2012). Based on photometric and spectroscopic observations with Spitzer, Chen et al. (2014) fitted the observed spectral shape of the excess using two blackbody components with temperatures of 237 and $91 \mathrm{~K}$. This two-component debris disk has a total fractional luminosity of $1.1 \times 10^{-4}$.

- The W4 band excess detection at star 59 (HD 152369) also seems to be solid.

None of those stars shows signs of accretion in our FEROS spectra. We ran a similar analysis on 221 random TGAS stars located at the same distance. None of those random stars showed consistent infrared excess as significant as our cluster members, giving a good hint that those disk detections may be real.

\section{V1062 Sco MG and its relation to the Upper Centaurus Lupus association}

The newly detected object is a comoving group of young stars at the edge of the huge $\left(845 \mathrm{deg}^{2}\right)$ UCL association. The ages of the V1062 Sco MG members range from less than 10 to about $25 \mathrm{Myr}$. Some of the younger stars were observed by the ROSAT satellite and show X-ray activity. The distance of the group from the Sun is $175 \pm 7 \mathrm{pc}$. Within the UCL complex there are a number of areas where star formation has recently taken place or is still going on. These areas are related to dense molecular clouds in UCL and have been studied by a number of authors in recent decades. However, no evidence has been obtained for a compact moving group around the variable star V1062 Sco.

\footnotetext{
1 http://irsa.ipac.caltech.edu/data/SPITZER/Enhanced/ SEIP/docs/seip_explanatory_supplement_v3.pdf
} 


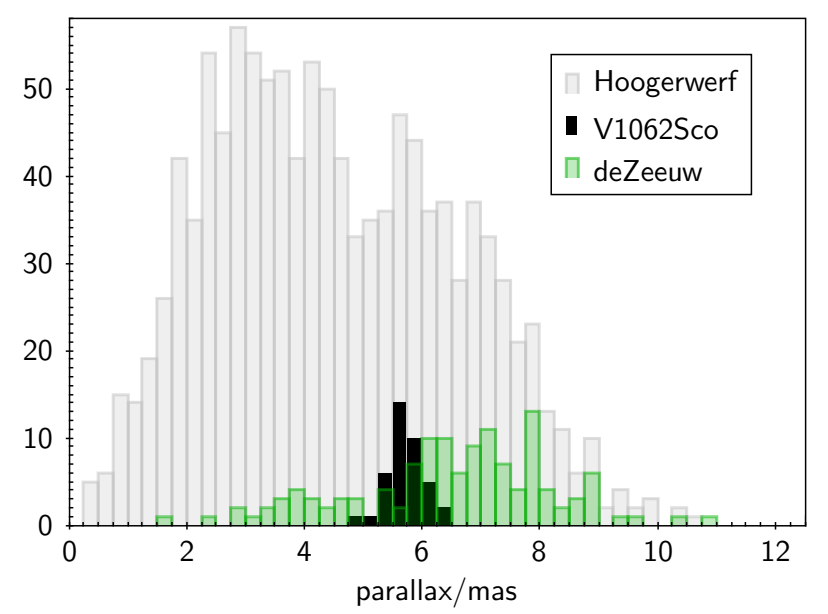

Fig. 7. Parallax distribution of stars in the UCL association. Grey bars show the TGAS parallaxes of the stars from Hoogerwerf (2000) and green bars show the HiPPARCOS stars from de Zeeuw et al. (1999). The black bars show the TGAS parallaxes of the 39 stars from Table 1, which are at least $\mathrm{m}(5 \mathrm{~d})$ members.

In his study of the Sco OB2_3 (UCL) area, Hoogerwerf (2000) searched for stars in the TRC catalogue (Hog et al. 1998), which was a predecessor of Tycho-2 and had proper motions consistent with the spatial velocity of the HIPPARCOS members of UCL from de Zeeuw et al. (1999). Using a convergent-point method, with further constraints on the proper motion distribution, magnitude and colour, Hoogerwerf found some 1200 candidate stars. Based on Monte Carlo simulations of the field star population, he expected a contamination of between 400 and 800 field stars in this sample. He thus concluded that, among the 1200 stars, a considerable number of association members should be present that are fainter than the about 150 HIPPARCOS stars from de Zeeuw et al. (1999).

In Fig. 6, we show Hoogerwerf stars as small grey dots in the background. These stars outline the extent of the UCL area. From the 35 stars in Table A.1 27 are listed in Hoogerwerf (2000), but only 6 are also contained in de Zeeuw et al. (1999). Also 6 stars in Table A.2 and 4 in Table A.3 are included in Hoogerwerf (2000). We crossmatched the UCL association candidates with TGAS, and show in Fig. 7 their distribution versus the trigonometric parallaxes. These candidates demonstrate a broad distribution with parallaxes between 1 and 10 mas. There is indicative of a bi-modal distribution with one part between 1 and 5 mas, probably the background field-star contaminants mentioned above, and between 5 and 8 mas of the UCL association proper. Also in Fig. 7 we show the distribution of the HIPPARCOS stars (but with TGAS parallaxes) from de Zeeuw et al. (1999). Their parallaxes are concentrated between 6 and 8 mas, showing a lower number of background contaminants. On top the parallax distributions in the UCL area in Fig. 7, we plot the distribution of TGAS parallaxes of our 39 stars from Table 1, which are at least m5d members. The V1062 Sco MG members show a narrow parallax distribution clearly indicating a sub-condensation at the periphery of the UCL complex.

Based on X-ray data from the ROSAT All-Sky-Survey, Krautter et al. (1997) discovered 136 new T Tauri stars in the Lupus SFR (their Tables 5, 6, and 11). The positions of these stars in UCL are also shown in Fig. 6. For $31 \mathrm{~T}$ Tauri stars in Krautter et al. (1997), we identified TGAS counterparts. In comparison to our V1062 Sco MV members, they show a much wider parallax spread of between 5 and 11 mas with a peak at 7.5 mas (ca. 133 pc).
Song et al. (2012) have spectroscopically identified 100 G-, $\mathrm{K}-$, and M-type members of the Scorpius-Centaurus complex. Sixteen of these are found in the UCL complex and are also plotted in Fig. 6. As already mentioned, their star SZB86 is an m(4d) kinematic member of our group (star 141). Song et al. (2012) derived ages of about $10 \mathrm{Myr}$ for stars in the Upper Centaurus Lupus region.

Galli et al. (2013) identified a comoving group with 109 premain-sequence stars and candidates in the Lupus SFR. Assuming that all stars share the same space motion, these authors derived individual parallaxes for stars with known RV and tentative parallaxes for the remaining group members. In their Fig. 3 they show the location of their UCL stars overplotted to the ${ }^{12} \mathrm{CO}$ intensity map from Tachihara et al. (2001). Galli et al. (2013) studied four subgroups called Lupus 1 to Lupus 4, which are all associated with strong ${ }^{12} \mathrm{CO}$ emission. The average kinematic distances of these subgroups range from 140 to 200 pc. Lupus 3, the most populated group at $(l, b)=\left(339^{\circ} .5,+9^{\circ} .5\right)$ in Fig. 6 has an average kinematic distance of $180 \mathrm{pc}$, but shows a considerable depth, i.e. the stars in Lupus 3 are located between 160 and $260 \mathrm{pc}$. Also the proper motions of this sample scatter between

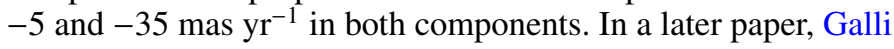
et al. (2015) derived masses and ages of the T Tauri star population in this SFR. The ages of their stars peak between 6 and $7 \mathrm{Myr}$, and are, on average, younger than ours.

Unlike the other SFRs in Lupus, which are embedded in molecular clouds and show considerable scatter in their proper motions, the V1062 Sco MG is concentrated in a distance range between 165 and $185 \mathrm{pc}$; the scatter of its proper motions is comparable to the uncertainties of the proper motions in TGAS, resp. HSOY, and, finally, it is not embedded in a molecular cloud on the Tachihara et al. (2001) ${ }^{12} \mathrm{CO}$ map. In Sect. 3.2 we found a moderate value for $E(B-V)$, i.e. an $A_{V}$ of about 0.2 mag in the direction of our group.

We additionally studied the interstellar-medium gas absorption in the NaI lines at 589.0 and $589.6 \mathrm{~nm}$ (Pascucci et al. 2015). Many of our targets show narrow-line absorption on top of the broad stellar absorption. These lines have a mean barycentric RV of $+0.5 \mathrm{~km} \mathrm{~s}^{-1}$ (in the LSR: $+6 \mathrm{~km} \mathrm{~s}^{-1}$ ) and have an $r m s$-spread of $2 \mathrm{~km} \mathrm{~s}^{-1}$. Over most of the cluster, the stellar and gas RVs are very similar. While the RV spread is random on the sky, the depth of the absorption line is noticeably strong (mean of $71 \%$ ) west of a great circle passing through $l, b=\left(343^{\circ},+4^{\circ}\right)$, an orientation of $-10^{\circ}$ ( $\mathrm{N}$ to $\mathrm{E}$ ), and small mean of $31 \%$ east of that line. This drop can be seen on the $\mathrm{H}_{\alpha}$ map of this velocity (HI4PI Collaboration et al. 2016). With these data, it is not possible to place the gas relative to the cluster and claim that the cluster still holds some of its original gas. We can however describe HI emission seen at other RV $\left(>+10\right.$ or $<-2 \mathrm{~km} \mathrm{~s}^{-1}$ in the LSR) by the HI4PI Collaboration et al. (2016) as background gas.

\section{Summary}

The Upper Centaurus Lupus (UCL) subgroup of the Sco-Cen OB-association contains a large number of some $130 \mathrm{~B}$ and $\mathrm{A}$ stars at a mean distance of $140 \mathrm{pc}$ from the Sun. Also it comprises a few subgroups, embedded in molecular clouds, where recent stars formation is going on. The new group around the variable star V1062 Sco is situated outside of these molecular clouds at a mean distance of $175 \pm 7 \mathrm{pc}$ from the Sun and contains 63 stars within a radius of $10 \mathrm{pc}$ around the centre. The stars in our moving group are not coeval, but show an age spread between 4 and $25 \mathrm{Myr}$ for which we have no explanation. This group is strongly comoving, as the $1 \mathrm{D}$ velocity dispersion is less than $1 \mathrm{~km} \mathrm{~s}^{-1}$. 
The 33 members from the TGAS catalogue are selected such that the difference between their kinematic and trigonometric parallaxes is smaller than the $1 \sigma$ error of this difference. For a subset of 12 stars, we could confirm a full 3D coincidence of their space motion with the mean space motion of the group. These definitely form a moving group or possibly an open cluster. For another subset of 27 stars the kinematically predicted parallaxes are confirmed by the trigonometric parallaxes from TGAS; they fulfil this necessary condition of being cluster members. Also 4 stars from HSOY have RVs confirmed. A last subset of 20 stars have predicted parallaxes consistent with the condition of co-movement. In total there are 63 stars belonging to this V1062 Sco MG. A possible contamination by field stars is about $3 \%$ estimated on the basis of the Besançon model of our Galaxy. In summary, we have clearly shown that the V1062 Sco MG is a new compact, young moving group within $200 \mathrm{pc}$ from the Sun. The masses of our 63 members range from 2.6 to $0.7 M_{\odot}$. With Gaia data release 2, announced for April 2018 we expect to reveal new and fainter members of the group.

Acknowledgements. This study was supported by Sonderforschungsbereich SFB 881 "The Milky Way System" (subprojects B5 and B7) of the German Research Foundation (DFG). This research has made use of the SIMBAD database and the VizieR catalogue access tool, operated at CDS, Strasbourg, France. This work has made use of data from the European Space Agency (ESA) mission Gaia (https: //www. cosmos.esa.int/gaia), processed by the Gaia Data Processing and Analysis Consortium (DPAC, https://www. cosmos.esa.int/web/ gaia/dpac/consortium). Funding for the DPAC has been provided by national institutions, in particular the institutions participating in the Gaia Multilateral Agreement. This publication makes use of VOSA, developed under the Spanish Virtual Observatory project supported from the Spanish MICINN through grant AyA2011-24052. This project has been supported by the Hungarian NKFI Grants K-115709 and K-119517 of the Hungarian National Research, Development and Innovation Office. A.D. was supported by the ÚNKP-17-4 New National Excellence Program of the Ministry of Human Capacities. AD would like to thank the City of Szombathely for support under Agreement No. 67.177-21/2016. B.G thanks Andres Jordan and Rafael Brahm for useful discussions and their help in reducing the FEROS data, as well as Caroline Bot and Jeroen Bouwman for their insights. The authors thank Ilaria Pascucci for her precious suggestions regarding IR excess and gas absorption. We would like to thank the anonymous referee for her/his very constructive and helpful comments.

\section{References}

Altmann, M., Roeser, S., Demleitner, M., Bastian, U., \& Schilbach, E. 2017, A\&A, 600, L4

Bayo, A., Rodrigo, C., Barrado Y Navascués, D., et al. 2008, A\&A, 492, 277 Blaauw, A. 1964, ARA\&A, 2, 213
Boller, T., Freyberg, M. J., Trümper, J., et al. 2016, A\&A, 588, A103 Brahm, R., Jordan, A., Hartman, J., \& Bakos, G. 2016, Astrophysics Source Code Library, [record ascl:1607.012]

Brahm, R., Hartman, J. D., Jordan, A., et al. 2018, AJ, 155, 112

Bressan, A., Marigo, P., Girardi, L., et al. 2012, MNRAS, 427, 127

Chen, C. H., Pecaut, M., Mamajek, E. E., Su, K. Y. L., \& Bitner, M. 2012, ApJ, 756,133

Chen, C. H., Mittal, T., Kuchner, M., et al. 2014, ApJS, 211, 25

Cieza, L. A., Schreiber, M. R., Romero, G. A., et al. 2010, ApJ, 712, 925

Cruz-Saenz de Miera, F., Chavez, M., Bertone, E., \& Vega, O. 2014, MNRAS, 437, 391

Cutri, R. M., Wright, E. L., Conrow, T. \& et al. 2012, VizieR Online Data Catalog: II $/ 311$

de Zeeuw, P. T., Hoogerwerf, R., de Bruijne, J. H. J., Brown, A. G. A., \& Blaauw, A. 1999, AJ, 117, 354

Fuhrmeister, B., \& Schmitt, J. H. M. M. 2003, A\&A, 403, 247

Gaia Collaboration (Brown, A. G. A., et al.) 2016, A\&A, 595, A2

Gaia Collaboration (van Leeuwen, F., et al.) 2017, A\&A, 601, A19

Galli, P. A. B., Bertout, C., Teixeira, R., \& Ducourant, C. 2013, A\&A, 558, A77

Galli, P. A. B., Bertout, C., Teixeira, R., \& Ducourant, C. 2015, A\&A, 580, A26

Gilmore, G., Randich, S., Asplund, M., et al. 2012, The Messenger, 147, 25

Goldman, B., Röser, S., Schilbach, E., et al. 2013, A\&A, 559, A43

Heske, A., \& Wendker, H. J. 1984, A\&AS, 57, 205

HI4PI Collaboration, Ben Bekhti, N., Flöer, L., et al. 2016, A\&A, 594, A116

Hog, E., Kuzmin, A., Bastian, U., et al. 1998, A\&A, 335, L65

Hoogerwerf, R. 2000, MNRAS, 313, 43

Jordi, C., Gebran, M., Carrasco, J. M., et al. 2010, A\&A, 523, A48

Kaufer, A., Stahl, O., Tubbesing, S., et al. 1999, The Messenger, 95, 8

Krautter, J., Wichmann, R., Schmitt, J. H. M. M., et al. 1997, A\&AS, 123, 329

Larson, R. B. 2002, in Modes of Star Formation and the Origin of Field Populations, eds. E. K. Grebel, \& W. Brandner, ASP Conf. Ser., 285, 442

Levato, H., Malaroda, S., Morrell, N., Solivella, G., \& Grosso, M. 1996, A\&AS, 118,231

Lindegren, L., Lammers, U., Bastian, U., et al. 2016, A\&A, 595, A4

Mamajek, E. E., Meyer, M. R., \& Liebert, J. 2002, AJ, 124, 1670

Mayor, M., Pepe, F., Queloz, D., et al. 2003, The Messenger, 114, 20

Michalik, D., Lindegren, L., \& Hobbs, D. 2015, A\&A, 574, A115

Monet, D. G., Levine, S. E., Canzian, B., et al. 2003, AJ, 125, 984

Morrissey, P., Conrow, T., Barlow, T. A., et al. 2007, ApJS, 173, 682

Oh, S., Price-Whelan, A. M., Hogg, D. W., Morton, T. D., \& Spergel, D. N. 2017, AJ, 153, 257

Pascucci, I., Edwards, S., Heyer, M., et al. 2015, ApJ, 814, 14

Randich, S., Tognelli, E., Jackson, R., et al. 2018, A\&A, 612, A99

Rizzuto, A. C., Ireland, M. J., \& Zucker, D. B. 2012, MNRAS, 421, L97

Röser, S., Schilbach, E., Piskunov, A. E., Kharchenko, N. V., \& Scholz, R.-D. 2011, A\&A, 531, A92

Röser, S., Schilbach, E., \& Goldman, B. 2016, A\&A, 595, A22

Santos, N. C., Sousa, S. G., Mortier, A., et al. 2013, A\&A, 556, A150

Skrutskie, M. F., Cutri, R. M., Stiening, R., et al. 2006, AJ, 131, 1163

Song, I., Zuckerman, B., \& Bessell, M. S. 2012, AJ, 144, 8

Tachihara, K., Toyoda, S., Onishi, T., et al. 2001, PASJ, 53, 1081

Zacharias, N., Finch, C., \& Frouard, J. 2017, AJ, 153, 166 
S. Röser et al.: A new compact young moving group around V1062 Scorpii

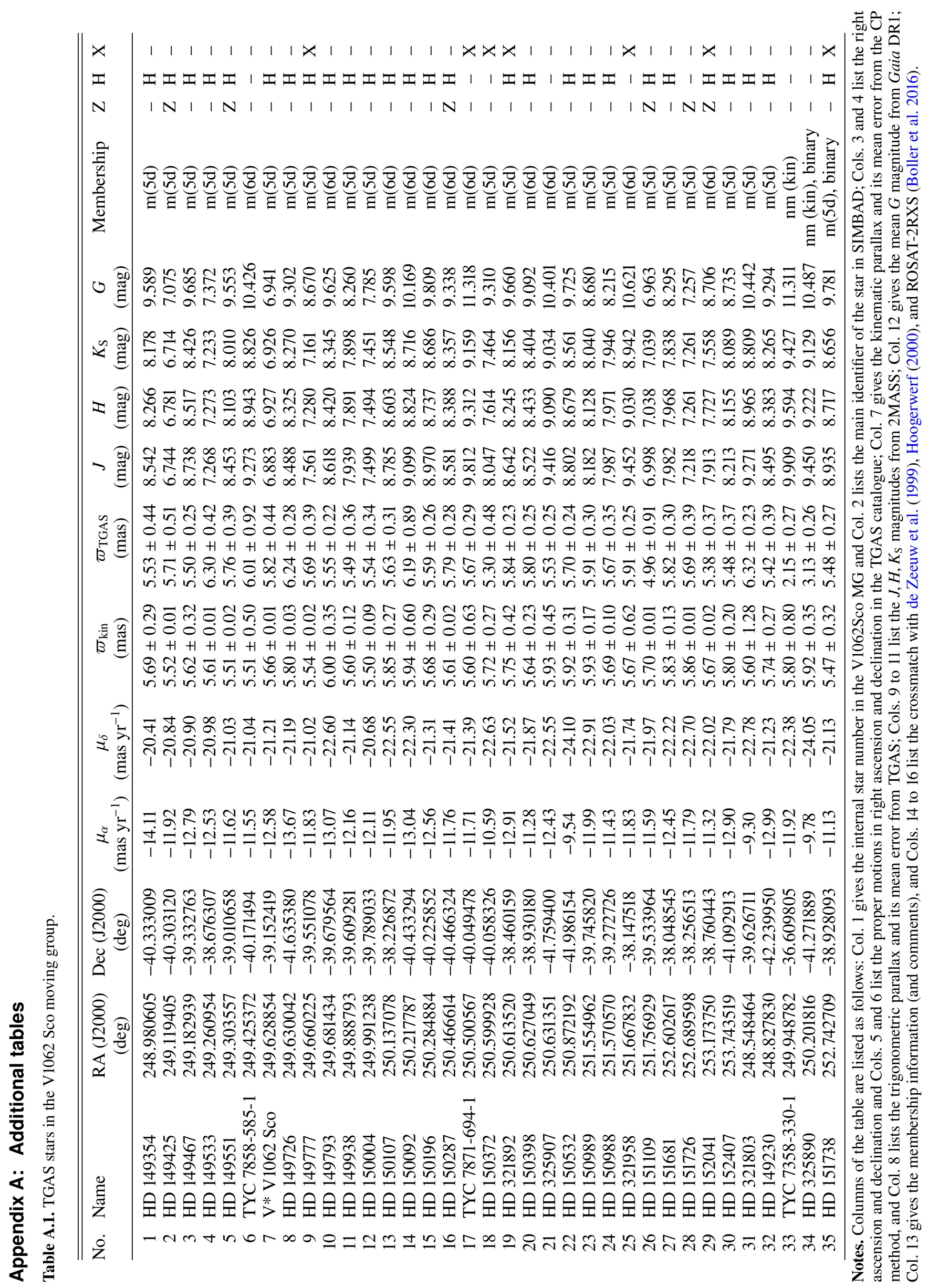

A81, page 9 of 13 
A\&A 614, A81 (2018)

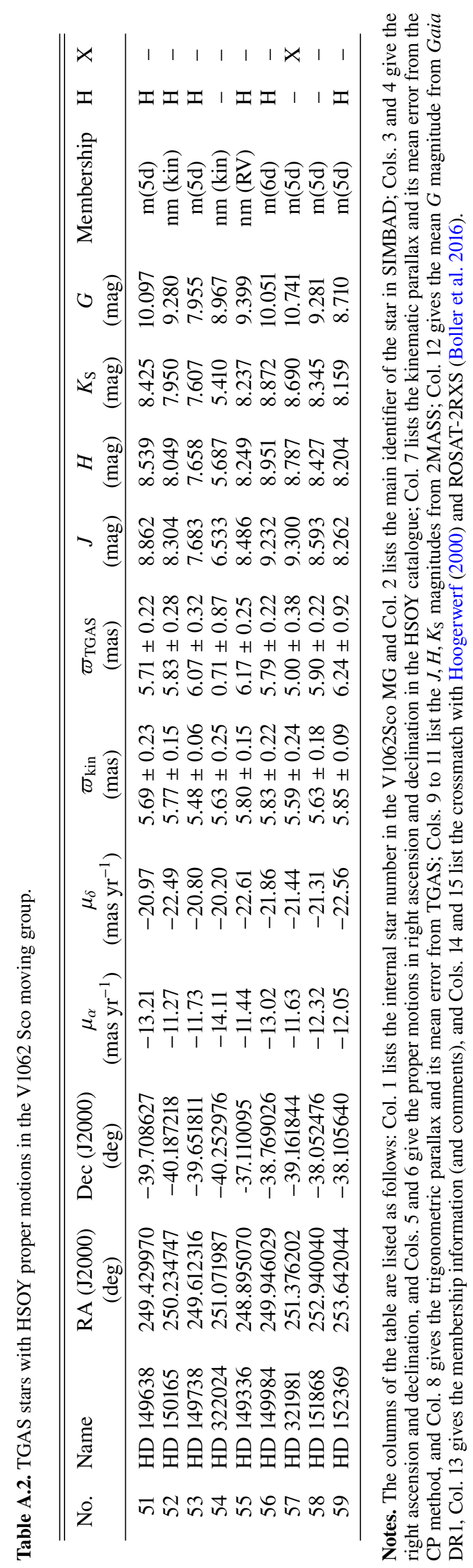




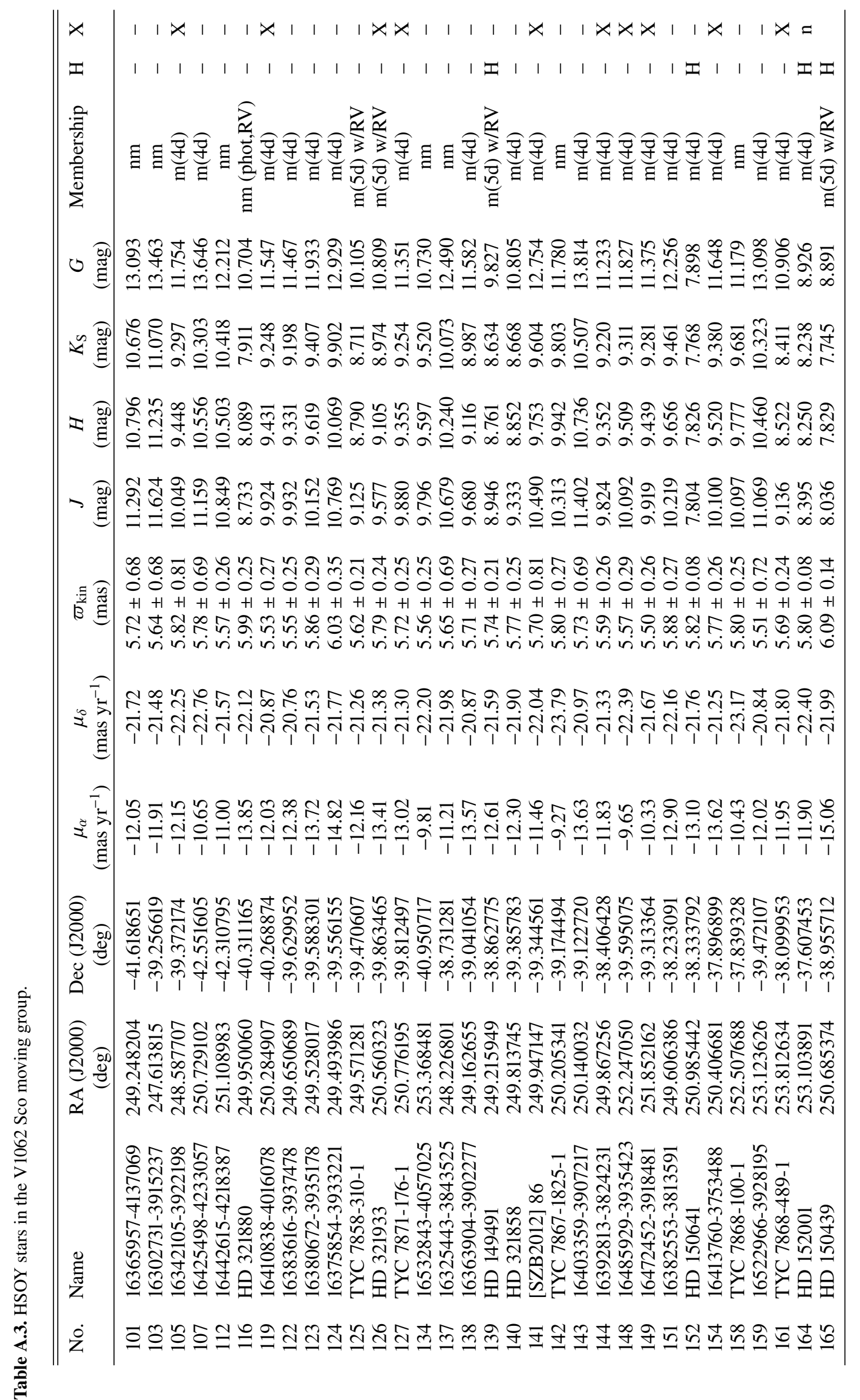


Table A.4. FEROS observation log.

\begin{tabular}{|c|c|c|c|c|c|c|}
\hline No. & MBJD & $\begin{array}{l}\text { Exp. time } \\
\text { (s) }\end{array}$ & $\begin{array}{r}S / N \\
(\text { at } 515 \mathrm{~nm}) \\
\end{array}$ & $\begin{array}{r}R V \\
\left(\mathrm{~km} \mathrm{~s}^{-1}\right) \\
\end{array}$ & Bisector & $\begin{array}{r}\text { CCF width } \\
(\AA)\end{array}$ \\
\hline 6 & 57952.08029 & 300 & 71 & $+3.61 \pm 0.21$ & $-1.755 \pm 0.011$ & 29.5 \\
\hline 10 & 57952.13738 & 300 & 130 & $+2.40 \pm 0.10$ & - & 23.4 \\
\hline 13 & 57952.17475 & 300 & 127 & $+1.53 \pm 0.07$ & - & 19.1 \\
\hline 14 & 57952.18116 & 300 & 89 & $+1.79 \pm 0.02$ & $0.052 \pm 0.010$ & 8.1 \\
\hline 15 & 57952.19783 & 300 & 108 & $+1.95 \pm 0.10$ & - & 20.6 \\
\hline 16 & 57952.20420 & 300 & 138 & $+3.64 \pm 0.31$ & $1.542 \pm 0.008$ & 28.8 \\
\hline 17 & 57952.21987 & 300 & 39 & $+2.41 \pm 0.04$ & - & 11.2 \\
\hline 17 & 57952.22880 & 600 & 50 & $+2.42 \pm 0.03$ & $-0.032 \pm 0.015$ & 11.2 \\
\hline 20 & 57953.11131 & 300 & 159 & $+2.49 \pm 0.10$ & - & 24.8 \\
\hline 21 & 57953.13064 & 300 & 79 & $+2.97 \pm 0.06$ & - & 14.8 \\
\hline 25 & 57953.17448 & 600 & 91 & $+1.03 \pm 0.02$ & $-0.115 \pm 0.010$ & 10.6 \\
\hline 25 & 57953.16868 & 300 & 62 & $+1.11 \pm 0.03$ & $-0.113 \pm 0.013$ & 10.7 \\
\hline 29 & 57953.21403 & 300 & 165 & $+2.07 \pm 0.36$ & $-0.118 \pm 0.007$ & 29.1 \\
\hline 34 & 57954.14386 & 300 & 82 & $-79.66 \pm 0.04$ & $0.047 \pm 0.011$ & 6.1 \\
\hline 34 & 57954.13572 & 300 & 79 & $-79.19 \pm 0.04$ & $0.158 \pm 0.011$ & 6.1 \\
\hline 34 & 57954.14792 & 300 & 79 & $-79.79 \pm 0.04$ & $0.142 \pm 0.011$ & 6.1 \\
\hline 34 & 57954.13980 & 300 & 82 & $-79.35 \pm 0.04$ & $0.106 \pm 0.011$ & 6.1 \\
\hline 35 & 57954.25678 & 300 & 73 & $+7.02 \pm 6.91$ & - & 46.6 \\
\hline 35 & 57954.26084 & 300 & 74 & $-0.77 \pm 6.78$ & $0.122 \pm 0.011$ & 46.9 \\
\hline 35 & 57954.25272 & 300 & 96 & $+7.60 \pm 5.56$ & $0.352 \pm 0.010$ & 47.5 \\
\hline 55 & 57954.04746 & 300 & 152 & $-33.30 \pm 0.02$ & $0.939 \pm 0.008$ & 10.4 \\
\hline 55 & 57954.04341 & 300 & 149 & $-33.23 \pm 0.02$ & - & 10.5 \\
\hline 55 & 57954.03935 & 300 & 143 & $-33.24 \pm 0.02$ & - & 10.5 \\
\hline 56 & 57954.09113 & 300 & 102 & $+1.74 \pm 0.05$ & - & 14.3 \\
\hline 56 & 57954.09521 & 300 & 105 & $+1.83 \pm 0.05$ & - & 14.3 \\
\hline 56 & 57954.09925 & 300 & 97 & $+1.73 \pm 0.05$ & $-0.202 \pm 0.010$ & 14.3 \\
\hline 116 & 57954.12032 & 300 & 52 & $-66.09 \pm 0.01$ & $0.003 \pm 0.014$ & 4.5 \\
\hline 116 & 57954.11217 & 300 & 53 & $-65.97 \pm 0.01$ & $-0.148 \pm 0.014$ & 4.6 \\
\hline 116 & 57954.11625 & 300 & 53 & $-66.06 \pm 0.01$ & $0.054 \pm 0.014$ & 4.5 \\
\hline 116 & 57954.10811 & 300 & 53 & $-66.08 \pm 0.01$ & $0.093 \pm 0.014$ & 4.5 \\
\hline 120 & 57954.22716 & 300 & 171 & $-46.60 \pm 0.01$ & $0.055 \pm 0.007$ & 5.2 \\
\hline 120 & 57954.22292 & 300 & 176 & $-46.60 \pm 0.01$ & $0.042 \pm 0.007$ & 5.2 \\
\hline 125 & 57954.08564 & 300 & 102 & $-3.18 \pm 0.01$ & $0.025 \pm 0.009$ & 4.4 \\
\hline 125 & 57954.08159 & 300 & 102 & $-3.15 \pm 0.01$ & $0.070 \pm 0.009$ & 4.4 \\
\hline 125 & 57954.07754 & 300 & 102 & $-3.14 \pm 0.01$ & $0.077 \pm 0.009$ & 4.4 \\
\hline 126 & 57954.18753 & 300 & 32 & $+6.43 \pm 0.02$ & $0.102 \pm 0.020$ & 5.9 \\
\hline 126 & 57954.17533 & 300 & 64 & $+6.21 \pm 0.01$ & $0.135 \pm 0.012$ & 6.0 \\
\hline 126 & 57954.17940 & 300 & 62 & $+6.29 \pm 0.01$ & $0.129 \pm 0.013$ & 6.1 \\
\hline 126 & 57954.18347 & 300 & 56 & $+6.22 \pm 0.01$ & $0.069 \pm 0.013$ & 6.1 \\
\hline 139 & 57954.06148 & 300 & 124 & $+5.13 \pm 0.06$ & $0.126 \pm 0.009$ & 16.6 \\
\hline 139 & 57954.05741 & 300 & 127 & $+5.10 \pm 0.06$ & - & 16.5 \\
\hline 139 & 57954.05336 & 300 & 127 & $+5.06 \pm 0.06$ & - & 16.5 \\
\hline 165 & 57954.20870 & 300 & 89 & $+1.62 \pm 0.11$ & - & 20.7 \\
\hline 165 & 57954.21700 & 300 & 118 & $+1.46 \pm 0.08$ & - & 20.8 \\
\hline 165 & 57954.21293 & 300 & 97 & $+1.51 \pm 0.10$ & - & 20.7 \\
\hline 165 & 57954.20464 & 300 & 79 & $+1.14 \pm 0.11$ & - & 20.6 \\
\hline
\end{tabular}

Notes. For each spectrum, we list the modified barycentric Julian Day, the integration time, $\mathrm{S} / \mathrm{N}$ at $515 \mathrm{~nm}$, RV, bisector value if it could be measured, and width of the CCF peak. 
S. Röser et al.: A new compact young moving group around V1062 Scorpii

Table A.5. Astrophysical parameters of member stars in the V1062 Sco moving group, followed by 4 rejected candidates.

\begin{tabular}{rccccc}
\hline \hline No. & $\begin{array}{c}R V \\
\left(\mathrm{~km} \mathrm{~s}^{-1}\right)\end{array}$ & $\begin{array}{c}T_{\text {eff }} \\
(\mathrm{K})\end{array}$ & $\log g$ & $\begin{array}{c}v \sin i \\
\left(\mathrm{~km} \mathrm{~s}^{-1}\right)\end{array}$ & {$[\mathrm{Fe} / \mathrm{H}]$} \\
\hline 6 & $+3.61 \pm 0.21$ & 6026 & 3.8 & 51.8 & +0.22 \\
10 & $+2.40 \pm 0.10$ & 6295 & 3.8 & 38.8 & -0.02 \\
13 & $+1.53 \pm 0.07$ & 6406 & 3.9 & 31.5 & +0.08 \\
14 & $+1.79 \pm 0.02$ & 5772 & 4.1 & 12.3 & +0.04 \\
15 & $+1.95 \pm 0.10$ & 6387 & 3.8 & 33.8 & +0.04 \\
16 & $+3.64 \pm 0.31$ & 6702 & 3.8 & 66.8 & +0.10 \\
17 & $+2.41 \pm 0.02$ & 5032 & 4.0 & 17.0 & +0.16 \\
20 & $+2.49 \pm 0.10$ & 5882 & 2.5 & 37.1 & -0.52 \\
21 & $+2.97 \pm 0.06$ & - & - & - & - \\
25 & $+1.06 \pm 0.02$ & 5332 & 4.2 & 16.7 & +0.05 \\
29 & $+2.07 \pm 0.36$ & 6197 & 4.0 & 79.2 & -0.04 \\
56 & $+1.77 \pm 0.03$ & 6144 & 4.0 & 23.5 & +0.04 \\
125 & $-3.16 \pm 0.01$ & 6254 & 4.3 & 4.9 & +0.03 \\
126 & $+6.26 \pm 0.01$ & 5336 & 4.2 & 8.1 & +0.03 \\
139 & $+5.10 \pm 0.03$ & 6383 & 3.9 & 27.5 & +0.01 \\
165 & $+1.45 \pm 0.05$ & 6204 & 3.9 & 33.2 & +0.08 \\
35 & - & 6020 & 3.5 & 119.9 & +0.15 \\
\hline 34 & $-79.50 \pm 0.02$ & 6790 & 3.6 & 8.8 & -0.52 \\
55 & $-33.26 \pm 0.01$ & 6508 & 4.3 & 17.0 & +0.17 \\
116 & $-66.05 \pm 0.01$ & 5022 & 2.7 & 5.0 & -0.37 \\
120 & $-46.60 \pm 0.00$ & - & - & - & - \\
\hline & & & & & \\
\hline
\end{tabular}

Notes. The measurements are weighted averages when multiple spectra were observed. In this case the uncertainties are the error on the mean. Radial velocities are from the CERES pipeline; stellar parameters are from ZASPE. 